\title{
3-Tesla MR spectroscopy in patients subjected to bone marrow transplantation: clinical correlations
}

\section{Spettroscopia-RM a 3 T nei pazienti sottoposti a trapianto di midollo osseo: correlazioni cliniche}

\author{
G. Sergiacomi • E. Gaspari • A. Taglieri • A. Meschini • V. Gisone • L. Cudillo • W. Arcese \\ G. Simonetti
}

\begin{abstract}
Dipartimento di Diagnostica per Immagini, Imaging Molecolare, Radiologia Interventistica e Radioterapia, Fondazione Policlinico Universitario “ Tor Vergata ”, Viale Oxford 81, 00133 Roma, Italy

Correspondence to: G. Sergiacomi, Tel.: +39-062-0902401, Fax: +39-062-0902404, e-mail: sergiacomi@ med.uniroma2.it
\end{abstract}

Received: 15 February 2011 / Accepted: 6 July 2011 / Published online: 10 February 2012

(C) Springer-Verlag 2012

\begin{abstract}
Purpose. This study evaluated the usefulness of 3-Tesla magnetic resonance (MR) spectroscopy in patients with non-Hodgkin's lymphoma (NHL) undergoing bone marrow transplantation (BMT).

Materials and methods. Twelve NHL patients who were candidates for BMT underwent three MR examinations of the lumbosacral spine: before ablative therapy for BMT, $15 \pm 4$ days and $54 \pm 24$ days after BMT. The MR study was supplemented by spectroscopic analysis. The lipid content was calculated and expressed as a percentage of lipid signal intensity relative to total signal intensity [fat fraction (FF)].

Results. In the first MR study, the FF was $62.5 \pm 7 \%$, in the second it was $70.75 \pm 5 \%$ and in the third it was $75 \pm 1 \%$. We observed a statistically significant difference between FF values calculated at the various MR studies $(p=0.02)$ and between red blood cell count ( $p=0.017$ ), platelet count $(p=0.003)$ and haematocrit $(p<0.001)$ at the three MR studies. FF had a statistically significant correlation with the number of circulating platelets $(p<0.01)$

Conclusions. MR spectroscopy of the bone marrow of NHL patients undergoing BMT is noninvasive and highly sensitive for characterising and monitoring bone marrow after BMT.
\end{abstract}

Keywords Bone marrow transplantation · 3-Tesla magnetic resonance $\cdot$ Spectroscopy

\begin{abstract}
Riassunto
Obiettivo. Scopo del presente lavoro è stato valutare l'utilità della spettroscopia mediante risonanza magnetica (RM) a 3 tesla nei pazienti affetti da linfoma non-Hodgkin (LNH) sottoposti a trapianto di midollo osseo (TMO). Materiali e metodi. Dodici pazienti affetti da LNH candidati al TMO sono stati sottoposti a 3 esami RM del rachide lombo-sacrale: prima della terapia ablativa per il TMO, dopo $15 \pm 4$ giorni dal TMO e dopo $54 \pm 24$ giorni. Lo studio RM è stato integrato con analisi spettroscopica. $\grave{E}$ stato calcolato il contenuto di lipidi, espresso in percentuale come intensità di segnale relativa dei lipidi rispetto all'intensità di segnale totale (fat fraction, FF).

Risultati. Nel primo studio RM si riscontrava una $F F=62,5 \% \pm 7 \%$; nel secondo una $F F=70,75 \% \pm 5 \%$; nel terzo una $F F=75 \% \pm 1 \%$. Abbiamo osservato una differenza statisticamente significativa tra $i$ valori di $F F$ calcolati ai vari controlli $R M(p=0,02)$, tra il numero dei globuli rossi $(p=0,017)$, delle piastrine $(p=0,003)$ e tra $i$ valori dell'ematocrito $(p<0,001)$ tra i tre controlli $R M$. La $F F$ presenta una correlazione statisticamente significativa con il numero delle piastrine circolanti $(p<0,01)$.

Conclusioni. La RM con studio spettroscopico del midollo osseo su pazienti con LNH sottoposti a TMO rappresenta una metodica non invasiva e molto sensibile nella caratterizzazione e monitoraggio del midollo osseo dopo TMO.
\end{abstract}

Parole chiave Trapianto di midollo osseo $\cdot$ Risonanza magnetica (RM) a 3 tesla $\cdot$ Spettroscopia 


\section{Introduction}

Transplantation of haematopoietic stem cells, or bone marrow transplantation (BMT), is considered a powerful therapeutic aid for treating many haematological and lymphatic diseases, as it enables the use of higher doses of cytotoxic chemotherapy agents in combination with radiotherapy [1-5]. Regardless of the type of transplant, the posttransplant course may often be affected by a series of complications, such as opportunistic infections, slow marrow repopulation and recurrence or graft-versus-host disease (GVHD). Autologous and peripheral stem-cell transplantation may substantially reduce the incidence of such events.

Monitoring marrow recovery following BMT is therefore an essential step in managing these patients [6, 7] and is usually done with a full blood count on peripheral blood, iliac crest biopsy and bone marrow aspiration. However, in addition to being invasive and painful, the latter procedures are carried out on a single location and therefore often fail to provide information regarding the entire haematopoietic system and cellularity and composition of the bone marrow. Magnetic resonance (MR) spectroscopy of the bone marrow, correlated with the pre- and posttransplant blood parameters, can provide information on the composition and possible physiological and pathological changes in vertebrae bone marrow before, during and after treatment. In parallel, MR study can confirm whether or not a local biopsy is truly representative of bone marrow status [6].

The main aim of our study was to analyse changes in certain specific MR spectroscopy parameters in patients affected non-Hodgkin's lymphoma (NHL) subjected to autologous BMT by means of follow-up examinations and to correlate such changes with blood parameters and specific characteristics of the transplant in order to assess the usefulness of follow-up with 3-Tesla MR imaging.

\section{Materials and methods}

Patient selection

All patients were informed about the experimental nature of the examination and provided prior written informed consent. The study was approved by the local ethics committee. We studied 12 men (mean age $35 \pm 7.2$ years) affected by NHL who were candidates for autologous BMT and 12 age- and sex-matched controls. Only individuals without contraindications underwent MR imaging of the lumbosacral spine. Each patient underwent three lumbosacral MR

\section{Introduzione}

Il trapianto di cellule staminali emopoietiche (TMO) è ora considerato un importante ausilio terapeutico per il trattamento di molte emolinfopatie, permettendo l'utilizzo di più alte dosi di farmaci chemioterapici citotossici in combinazione con la radioterapia [1-5]. Indipendentemente dalla tipologia di trapianto, il decorso post-trapianto può essere spesso influenzato da una serie di complicanze quali infezioni opportunistiche, una lenta ripopolazione midollare, comparsa di recidive e la reazione contro l'ospite (graftversus-host disease, GVHD); il trapianto autologo di cellule staminali e di cellule staminali periferiche può notevolmente ridurre l'incidenza di tali eventi.

Pertanto, il monitoraggio della ripresa midollare del paziente nel periodo successivo al TMO rappresenta un momento essenziale nella gestione di questi pazienti [6, 7]. Tale monitoraggio è solitamente effettuato mediante esame emocromocitometrico su sangue periferico, biopsia della cresta iliaca e aspirato midollare. Quest'ultime oltre ad essere procedure invasive e dolorose, essendo ottenute da una singola localizzazione, spesso non forniscono dati rappresentativi dell'intero sistema ematopoietico, della cellularità e della composizione del midollo osseo. La risonanza magnetica (RM) con indagine spettroscopica (MRS) del midollo osseo, correlata con i parametri ematologici pre- e post-trapianto di cellule staminali emopoietiche consente di ottenere, in maniera non invasiva, informazioni sulla composizione e sulle eventuali alterazioni fisiologiche e patologiche del midollo osseo presenti nelle vertebre prima, durante e dopo il trattamento. Parallelamente lo studio RM può confermare se una biopsia locale è in grado, o meno di essere rappresentativa del reale stato riguardo al midollo osseo [6].

L'obiettivo principale del nostro studio è stato quello di analizzare le variazioni espresse da determinati e specifici parametri di MRS nei pazienti affetti da linfoma nonHodgkin (LNH) sottoposti a trapianto autologo di cellule staminali emopoietiche effettuando controlli a distanza e correlandoli con i parametri e le caratteristiche specifiche del trapianto al fine di poter stabilire l'utilità del follow-up mediante esame RM a 3 tesla.

\section{Materiali e metodi}

Selezione dei pazienti

Tutti i pazienti sono stati informati sul carattere sperimentale dell'esame ed hanno firmato un consenso informato prima di essere sottoposti all'indagine. Lo studio è stato approvato dal comitato etico interno. Sono stati studiati un totale di

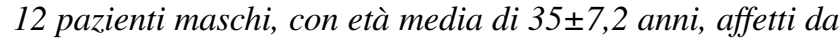
LNH candidati al TMO autologo, confrontati con 12 soggetti di controllo concordi per età e sesso. I soggetti che non presentavano controindicazioni sono stati sottoposti a esame RM del rachide lombo-sacrale. Ogni paziente ha eseguito 3 
examinations: the first immediately prior to ablative therapy, the second approximately 15 days after BMT and the third approximately 3 months after BMT.

\section{Image acquisition}

Morphological and metabolic spectroscopy images were acquired with a 3-Tesla unit (Philips Intera Achieva, Best, The Netherlands) equipped with maximum gradient amplitude and slew rate of $80 \mathrm{mT} / \mathrm{m}$ and $200 \mathrm{mT} / \mathrm{m} / \mathrm{ms}$, respectively; we used body coils for signal transmission and synergy spine phased-array coils for signal reception. The lumbar spine was studied using the following sequences: T2 turbo spin echo (TSE), including the entire lumbar-sacral segment in the acquisition volume [time to repetition (TR) 5,086 ms, time to echo (TE) $120 \mathrm{~ms}$; T1-weighted TSE (TR $265 \mathrm{~ms}$, TE $7.2 \mathrm{~ms}$ ); T2-weighted TSE with selective fat signal suppression [short-tau inversion recovery (STIR)] to assess the presence of spongy bone oedema, which is an indication of recent fracture for collapsed vertebral bodies or of neoplastic replacement as regards the vertebral bodies involved by disease. All sequences were acquired in the sagittal plane with a slice thickness of $4 \mathrm{~mm}$ and interslice gap of $0.4 \mathrm{~mm}$; field of view was $300 \mathrm{~mm}$, with a matrix of $352 \times 280$. On the basis of the T2-weighted, especially STIR, images, two independent observers identified the midlumbar vertebrae with normal signal intensity, which were selected for spectroscopic acquisition volume placement. Acquisition was performed with a 2D double spin-echo point-resolved spatially localised spectroscopic sequence (PRESS), with single voxel analysis (TR 2,000 ms, TE $40 \mathrm{~ms}$, spectral amplitude 2,000 Hz, matrix $512 \times 512$, nominal voxel resolution $15 \times 15 \times 15 \mathrm{~mm}$ ). Field homogeneity was adjusted by a localised 3D automatic shimming procedure. Acquisition time for the spectroscopic sequence was 2 min on average. Spectroscopic acquisition was used to assess the curves for water and fat.

The volume of interest (VOI) was selected on the basis of sagittal T2-weighted and T2-STIR images taking care to select a homogeneous volume of vertebral body. In the 12 healthy controls and in NHL patients without vertebral collapse, a spectroscopic acquisition was performed with a duration of $2.12 \mathrm{~min}$, with a VOI placed at the level of L3; if L3 was collapsed or diseased, the VOI was placed over L2 or L4 (Fig. 1). To assess intra- and interobserver variability, for each examination, the operators independently selected two VOI on the same vertebra and recorded results of the spectroscopic analysis. Spectra obtained were processed by Fourier transformation. Fat content was expressed as a percentage of fat signal intensity relative to total signal intensity [fat fraction (FF)]. controlli di RM del rachide lombo-sacrale: il primo controllo subito prima dell'inizio della terapia ablativa per il TMO, il secondo a distanza di circa quindici giorni dall'esecuzione del TMO e il terzo dopo circa tre mesi.

Acquisizione delle immagini

Le immagini morfologiche, così come quelle metaboliche di spettroscopia, sono state acquisite con un'apparecchiatura ad alto campo da $3 \mathrm{~T}$ (Philips Intera Achieva, Best, Paesi Bassi) equipaggiata con gradienti di ampiezza massima e tempo di salita di $80 \mathrm{mT} / \mathrm{m}$ e $200 \mathrm{mT} / \mathrm{m} / \mathrm{ms}$ rispettivamente, utilizzando la bobina del corpo per la trasmissione degli impulsi di eccitazione e delle bobine sinergy spine phasedarray per la ricezione del segnale. Lo studio del rachide lombare è stato eseguito mediante le seguenti sequenze: T2-turbo spin echo (TSE), includendo nel volume d'acquisizione l'intero tratto lombo-sacrale [tempo di ripetizione (TR) $5086 \mathrm{~ms}$, tempo di eco (TE) $120 \mathrm{~ms}$ ]; TSE T1 pesata (TR $265 \mathrm{~ms}$, TE 7,2 ms); TSE T2 pesata con soppressione selettiva del segnale del tessuto adiposo (short-tau inversion recovery, STIR) al fine di valutare l'eventuale presenza di edema intraspongioso, indice di frattura recente per $i$ somi vertebrali crollati o di sostituzione neoplastica per quanto riguarda i somi vertebrali sede di localizzazione di malattia. Tutte le sequenze sono state acquisite sul piano sagittale con spessore di strato di $4 \mathrm{~mm}$ ed intervallo tra le sezioni di $0,4 \mathrm{~mm}$; il campo di vista (FOV) era di $300 \mathrm{~mm}$ con una matrice di $352 \times 280$. Sulla base delle immagini $T 2$ pesate, soprattutto STIR, sono state individuate da due osservatori indipendenti le vertebre a livello medio-lombare con normale intensità di segnale, selezionate per il successivo posizionamento del volume di acquisizione spettroscopica. L'acquisizione è stata eseguita con sequenza double spin-echo point-resolved spatially localized spectroscopic sequence (PRESS) con tecnica bidimensionale, con analisi single voxel (TR $2000 \mathrm{~ms}, T E 40 \mathrm{~ms}$, ampiezza spettrale $2000 \mathrm{~Hz}$, matrice di $512 \times 512$, risoluzione nominale del voxel di $15 \mathrm{~mm} \times 15 \mathrm{~mm} \times 15 \mathrm{~mm}$ ). L'omogeneizzazione del campo magnetico è stata compiuta con una procedura automatica di shimming tridimensionale localizzato. Il tempo di acquisizione della sequenza spettroscopica è stato in media di due minuti. Con l'acquisizione spettroscopica sono stati valutati le curve corrispondenti all'acqua e ai lipidi.

Il volume d'interesse sottoposto ad analisi spettroscopica (VOI) è stato selezionato sulla base delle immagini T2pesate e T2-STIR sul piano sagittale, cercando di scegliere un volume di corpo vertebrale il più possibile omogeneo. Nei 12 pazienti sani e in quelli affetti da LNH senza crollo vertebrale è stata eseguita un'acquisizione spettroscopica, della durata di 2,12 minuti, con VOI posto a livello del corpo vertebrale di L3; se quest'ultimo era crollato o sede di malattia si è scelto di disporre il VOI su L2 o su L4 (Fig. 1). Al fine di valutare la variabilità intra-osservatore e inter-osservatore, ciascun operatore, indipendentemente dall'altro, ha selezionato per ogni esame 2 VOI sulla medesima vertebra e registrato i risultati dell'analisi spettroscopica. Gli spettri otte- 


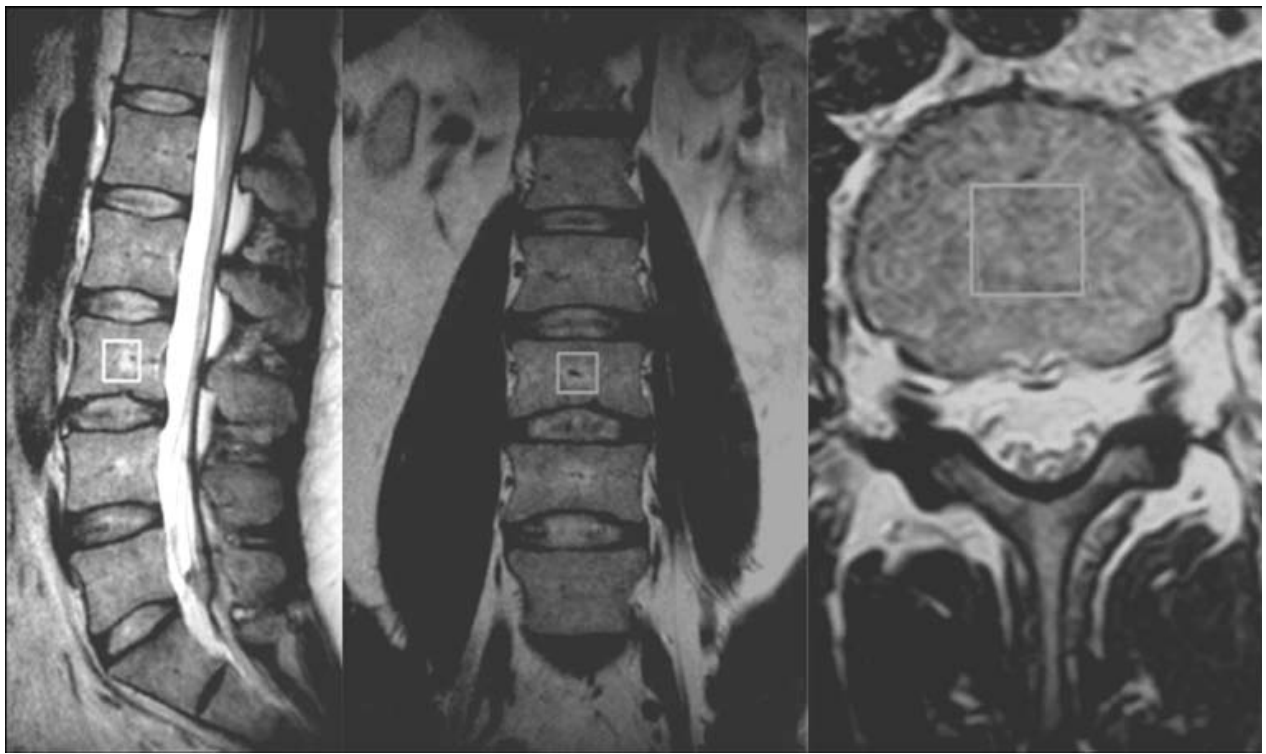

Fig. 1 Voxel positioning for spectroscopy within the vertebral body of L3 in a healthy control.

Fig. 1 Esempio di posizionamento del voxel per la spettroscopia all'interno del soma vertebrale di L3 in un paziente di controllo.

Statistical analysis

Each observer analysed 72 spectroscopies for a total of 144 observations. FF values recorded by the two observers for each patient at the same time points (T0, T1, T2) were matched to create a Bland-Altman plot [8], and Lin's method $[9,10]$ was used to calculate the interobserver coefficient of concordance (Rho-C) and the limits of agreement. Similarly, FF pair values recorded by each observer for the same patient at the same time (T0, T1, T2) were used to create a Bland-Altman plot was for each observer, and limits of agreement and repeatability coefficient were calculated. FF and blood count values were compared with each other at the various time points by means of an analysis of variance (ANOVA) test. FF was then correlated to blood count values. A value of $p<0.05$ was considered to indicate statistical significance.

\section{Results}

Data obtained were assessed by two radiologists and one haematologist working in consensus (GS, AM, LC). The first MR examination was performed in all patients before the start of ablative therapy and stem cell infusion; the second was carried out $15 \pm 4$ days after BMT and the third $54 \pm 24$ days after BMT (Figs. 2-4). At the first MR examination, the red blood cell (RBC) count was $4.29 \pm 0.3$ million $/ \mathrm{mm}^{3}$, platelet count $194 \pm 34$ thousand $/ \mathrm{mm}^{3}$ and white blood cell (WBC) count $6.4 \pm 4.7$ thousand $/ \mathrm{mm}^{3}$, with a mean neutrophil count of $5 \pm 4.9$ thousand $/ \mathrm{mm}^{3}$ and a lymphocyte count $0.71 \pm 0.31$ thousand $/ \mathrm{mm}^{3}$. Haematocrit was nuti sono stati elaborati mediante la trasformata di Fourier. Il contenuto di lipidi è stato espresso in percentuale come intensità di segnale relativa dei lipidi rispetto all'intensità di segnale totale (fat fraction, $F F$ ).

Analisi statistica

Ciascun osservatore ha analizzato 72 spettroscopie per un totale di 144 osservazioni. Appaiando $i$ valori di FF rilevati dai due osservatori per ciascun paziente nel medesimo tempo $T(T 0, T 1, T 2)$ è stato realizzato un grafico di Bland-Altman [8] e, mediante il medoto di Lin [9, 10], è stato calcolato il coefficiente di concordanza inter-osservatore (Rho_C) ed i limiti di accordo. Analogamente, appaiando le coppie di valori di FF rilevati da ciascuno dei due osservatori per lo stesso paziente, nel medesimo tempo $T(T 0, T 1, T 2)$, è stato costruito un grafico di Bland-Altman per ciascun osservatore e sono stati calcolati i limiti di accordo ed il coefficiente di ripetibilità. I valori di FF e quelli emocromocitometrici sono stati confrontati tra loro nei vari controlli mediante un test di analisi della varianza (ANOVA). La FF è poi stata correlata con $i$ valori emocromocitometrici. È stato considerato significativo ai fini statistici un valore di p inferiore a 0,05.

\section{Risultati}

I dati ottenuti sono stati valutati rispettivamente da due radiologi e un ematologo in consenso (G.S., A.M. e L.C.). La prima RM è stata eseguita in tutti i pazienti prima dell'inizio della terapia ablativa e dell'infusione delle cellule; la seconda RM è stata eseguita in media dopo $15 \pm 4$ giorni e la terza RM dopo $54 \pm 24$ giorni dal trapianto di midollo osseo (Figg. 2-4). L'emocromo ha mostrato un numero di globuli rossi alla prima $R M$ di $4,29 \pm 0,3 \times 10^{6} / \mathrm{mm}^{3}$, di piastrine di 


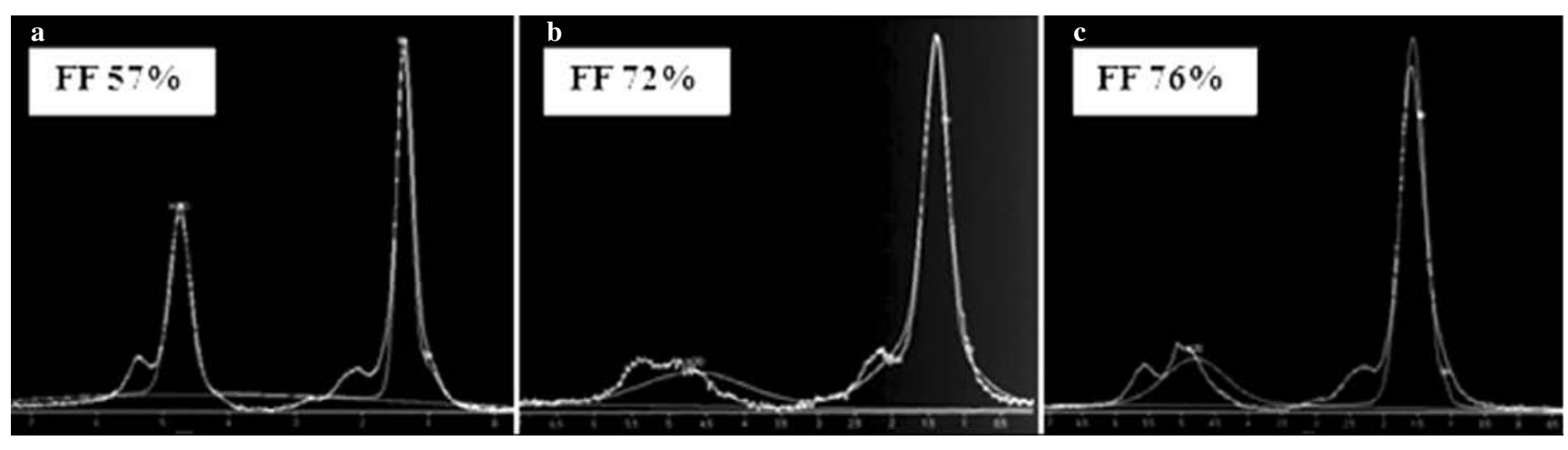

Fig. 2a-c A 46-year-old man affected by non-Hodgkin's lymphoma. Spectroscopy before (a) and 11 (b) and 84 (c) days after BMT shows net increase of FF\% associated with decrease in water peak at the second and third follow-up examination, indicating a failed bone marrow recovery (platelet count at the third follow-up was $30 \times 10^{3} / \mathrm{mm}^{3}$ ).

Fig. 2a-c Paziente di 46 anni affetto da LNH. Lo spettro ottenuto prima del TMO (a), dopo 11 giorni (b) e dopo 84 giorni dal TMO (c) mostra un netto aumento della FF\% con decremento del picco idrico al II ed al III controllo, indice di un mancato recupero midollare (piastrine al III controllo $30 \times 10^{3} / \mathrm{mm}^{3}$ ).
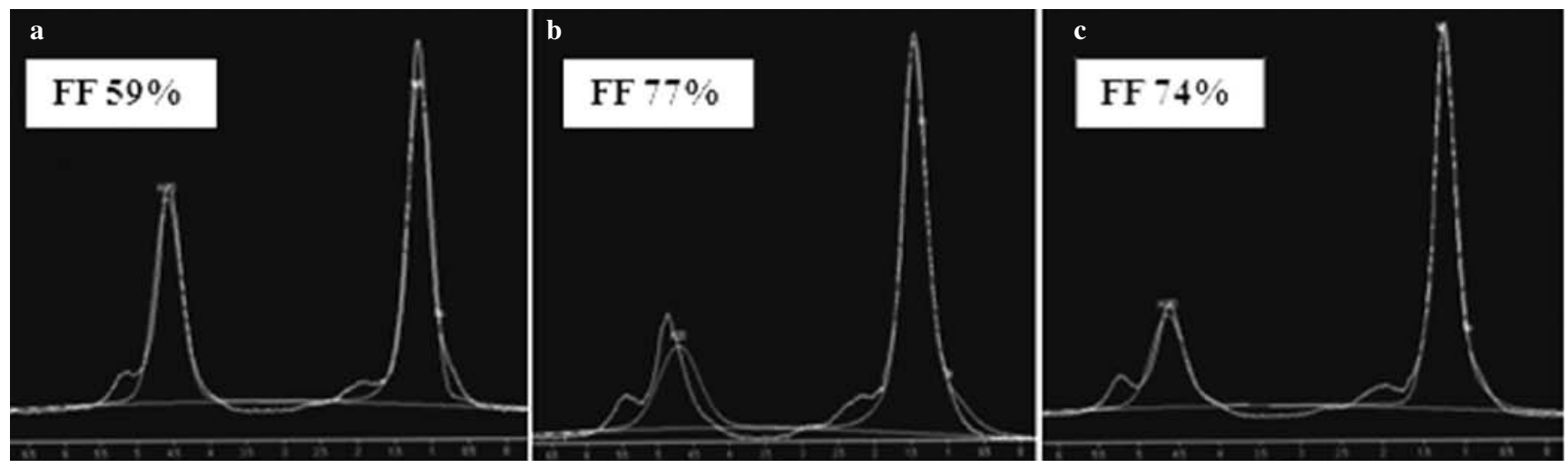

Fig. 3a-c A 34-year-old man affected by non-Hodgkin's lymphoma. Spectroscopy before (a) and 17 (b) and 64 (c) days after BMT shows failed recovery of water peak with increase of FF\% at the second and third follow-up examinations, indicating a failed bone marrow recovery (platelet count at the third follow-up examination was $\left.45 \times 10^{3} / \mathrm{mm}^{3}\right)$.

Fig. 3a-c Paziente di 34 anni affetto da LNH. Lo spettro ottenuto prima del TMO (a), dopo 17 giorni (b) e dopo 64 giorni dal TMO (c) mostra un mancato recupero del picco idrico con aumento della $\mathrm{FF} \%$ al II ed al III controllo, come per mancato recupero midollare (piastrine al III controllo $45 \times 10^{3} / \mathrm{mm}^{3}$ ).
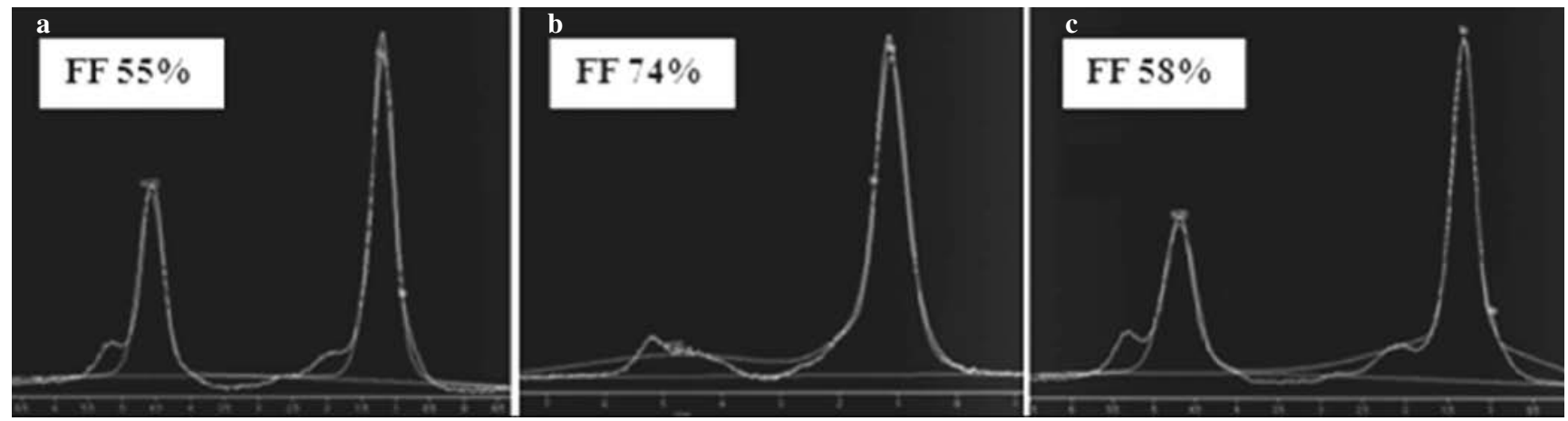

Fig. 4a-c A 27-year-old man affected by non-Hodgkin's lymphoma. Spectroscopy before (a) and 11 (b) and 79 (c) days after BMT shows decreased water peak at the second follow-up examination, with increase at the third examination, indicating bone marrow recovery (platelet count at the third follow-up was $\left.155 \times 10^{3} / \mathrm{mm}^{3}\right)$.

Fig. 4a-c Paziente di 27 anni affetto da LNH. Lo spettro ottenuto prima del TMO (a), dopo 11 giorni (b) e dopo 79 giorni dal TMO (c) mostra un decremento del picco idrico al II controllo con completo ripristino al III controllo, indice di un buon recupero midollare (piastrine al III controllo $155 \times 10^{3} / \mathrm{mm}^{3}$ ). 
Table 1 Blood values before and after bone marrow transplantation (BMT)

\begin{tabular}{lllll}
\hline & 1st examination & 2nd examination & 3rd examination & $p$ value* \\
\hline Days from BMT & 0 & $15 \pm 4$ days & $54 \pm 24$ days & - \\
Red blood cells & $4.29 \pm 0.3$ million $/ \mathrm{mm}^{3}$ & $3.5 \pm 0.5$ million $/ \mathrm{mm}^{3}$ & $3.4 \pm 0.9 \mathrm{million} / \mathrm{mm}^{3}$ & 0.017 \\
Platelets & $194 \pm 34$ thousand $/ \mathrm{mm}^{3}$ & $104 \mathrm{z} \pm 132$ thousand $/ \mathrm{mm}^{3}$ & $37 \pm 20$ thousand $/ \mathrm{mm}^{3}$ & 0.003 \\
White blood cells & $6.4 \pm 4.7$ thousand $/ \mathrm{mm}^{3}$ & $7.2 \pm 5.2$ thousand $/ \mathrm{mm}^{3}$ & $6.2 \pm 8$ thousand $/ \mathrm{mm}^{3}$ & 0.63 \\
Neutrophils & $5 \pm 4.9$ thousand $/ \mathrm{mm}^{3}$ & $6.32 \pm 4.7$ thousand $/ \mathrm{mm}^{3}$ & $5 \pm 7.5$ thousand $/ \mathrm{mm}^{3}$ & 0.22 \\
Lymphocytes & $0.71 \pm 0.31$ thousand $/ \mathrm{mm}^{3}$ & $0.22 \pm 0.12$ thousand $/ \mathrm{mm}^{3}$ & $0.62 \pm 0.4$ thousand $/ \mathrm{mm}^{3}$ & 0.22 \\
Ht (\%) & $37 \pm 6$ & $29 \pm 2.4$ & $29 \pm 5$ & $<0.001$ \\
FF $(\%)$ & $62.5 \pm 7$ & $70.75 \pm 5$ & $75 \pm 1$ & 0.02 \\
\hline
\end{tabular}

$H t$, haematocrit; $F F$, fat fraction; $B M T$, bone marrow transplantation

*Analysis of variance

Tabella 1 Valori ematici pre- e post-trapianto di midollo osseo (TMO)

\begin{tabular}{lllll}
\hline & I controllo & II controllo & III controllo & $p^{*}$ \\
\hline Giorni dal TMO & 0 giorni & $15 \pm 4$ & $54 \pm 24$ & - \\
Globuli rossi & $4,29 \pm 0,3 \times 10^{6} / \mathrm{mm}^{3}$ & $3,5 \pm 0,5 \times 10^{6} / \mathrm{mm}^{3}$ & $3,4 \pm 0,9 \times 10^{6} / \mathrm{mm}^{3}$ & 0,017 \\
Piastrine & $194 \pm 34 \times 10^{3} / \mathrm{mm}^{3}$ & $104 \pm 132 \times 10^{3} / \mathrm{mm}^{3}$ & $37 \pm 20 \times 10^{3} / \mathrm{mm}^{3}$ & 0,003 \\
Globuli bianchi & $6,4 \pm 4,7 \times 10^{3} / \mathrm{mm}^{3}$ & $7,2 \pm 5,2 \times 10^{3} / \mathrm{mm}^{3}$ & $6,2 \pm 8 \times 10^{3} / \mathrm{mm}^{3}$ & 0,63 \\
Neutrofili & $5 \pm 4,9 \times 10^{3} / \mathrm{mm}^{3}$ & $6,32 \pm 4,7 \times 10^{3} / \mathrm{mm}^{3}$ & $5 \pm 7,5 \times 10^{3} / \mathrm{mm}^{3}$ & 0,22 \\
Linfociti & $0,71 \pm 0,31 \times 10^{3} / \mathrm{mm}^{3}$ & $0,22 \pm 0,12 \times 10^{3} / \mathrm{mm}^{3}$ & $0,62 \pm 0,4 \times 10^{3} / \mathrm{mm}^{3}$ & 0,22 \\
Ht $(\%)$ & $37 \pm 6$ & $29 \pm 2,4$ & $29 \pm 5$ & $<0,001$ \\
FF $(\%)$ & $62,5 \pm 7$ & $70,75 \pm 5$ & $75 \pm 1$ & 0,02 \\
\hline
\end{tabular}

$\mathrm{Ht}$, ematocrito; FF, fat fraction; TMO, trapianto di midollo osseo

*ANOVA test

$37 \pm 6 \%$ on average. $\mathrm{FF}$ at the first MR examination was $62.5 \pm 7 \%$. At the second MR examination, the RBC count was $3.5 \pm 0.5 \mathrm{million} / \mathrm{mm}^{3}$, platelet count $104 \pm 132$ thousand $/ \mathrm{mm}^{3}$ and WBC count was $7.2 \pm 5.2$ thousand $/ \mathrm{mm}^{3}$, with $6.32 \pm 4.7$ thousand $/ \mathrm{mm}^{3}$ neutrophils and $0.22 \pm 0.12$ thousand $/ \mathrm{mm}^{3}$ lymphocytes. Haematocrit was $29 \% \pm 2.4 \%$. $\mathrm{FF}$ at the second MR examination was $70.75 \% \pm 5 \%$. At the third MR examination, RBC count was $3.4 \pm 0.9$ million $/ \mathrm{mm}^{3}$, platelets $37 \pm 20$ thousand $/ \mathrm{mm}^{3}$ and $\mathrm{WBC}$ $6.2 \pm 8$ thousand $/ \mathrm{mm}^{3}$, with $5 \pm 7.5$ thousand $/ \mathrm{mm}^{3}$ neutrophils and $0.62 \pm 0.4$ thousand $/ \mathrm{mm}^{3}$ lymphocytes. Haematocrit was $29 \% \pm 5 \%$. FF at the third MR examination was $75 \% \pm 1 \%$.

These was a statistically significant difference between FF calculated at the various time points $(p=0.02)$ between RBC $(p=0.017)$, platelet $(p=0.003)$ and haematocrit $(p<0.001)$ values, whereas no significant differences were found in the overall number of WBC ( $p=0.63)$, neutrophils $(p=0.22)$ and platelets $(p=0.22)$ between the three time points (Table 1). FF was significantly related to the number of platelets $(r=0.72 ; p=0.01)$, whereas no statistically significant correlation was found between FF and the number of RBC, WBC, neutrophils, lymphocytes and haematocrit (Fig. 5). The mean of the differences between FF record-
$194 \pm 34 \times 10^{3} / \mathrm{mm}^{3}$ e di globuli bianchi di $6,4 \pm 4,7 \times 10^{3} / \mathrm{mm}^{3}$, con un numero medio di neutrofili di $5 \pm 4,9 \times 10^{3} / \mathrm{mm}^{3}$ e di linfociti di $0,71 \pm 0,31 \times 10^{3} / \mathrm{mm}^{3}$. L'ematocrito era in media di $37 \% \pm 6 \%$. La FF alla prima RM era di $62,5 \% \pm 7 \%$. Alla seconda RM abbiamo osservato un numero di globuli rossi di $3,5 \pm 0,5 \times 10^{6} / \mathrm{mm}^{3}$, un numero di piastrine di $104 \pm 132 \times 10^{3} /$ $\mathrm{mm}^{3}$ e un numero di leucociti di $7,2 \pm 5,2 \times 10^{3} / \mathrm{mm}^{3}$, con $6,32 \pm 4,7 \times 10^{3} / \mathrm{mm}^{3}$ neutrofili e $0,22 \pm 0,12 \times 10^{3} / \mathrm{mm}^{3}$ linfociti. L'ematocrito ammontava al $29 \% \pm 2,4 \%$. La FF alla seconda $R M$ era di $70,75 \% \pm 5 \%$. Il terzo controllo $R M$ ha mostrato $3,4 \pm 0,9 \times 10^{6} / \mathrm{mm}^{3}$ di globuli rossi, $37 \pm 20 \times 10^{3} / \mathrm{mm}^{3}$ di piastrine e $6.2 \pm 8 \times 10^{3} / \mathrm{mm}^{3}$ di leucociti, con $5 \pm 7,5 \times 10^{3} / \mathrm{mm}^{3}$ neutrofili e $0,62 \pm 0,4 \times 10^{3} / \mathrm{mm}^{3}$ linfociti. L'ematocrito ammontava al $29 \% \pm 5 \%$. La FF alla terza RM era di $75 \% \pm 1 \%$.

Vi è una differenza statisticamente significativa tra $i$ valori percentuali di FF calcolati ai vari controlli $R M(p=0,02)$, tra il numero dei globuli rossi $(p=0,017)$, delle piastrine $(p=0,003)$ e tra $i$ valori dell'ematocrito $(p<0,001)$; mentre non si sono osservate differenze statisticamente significative nel numero dei globuli bianchi in toto $(p=0,63)$, dei neutrofili $(p=0,22)$ e delle piastrine $(p=0,22)$ tra i tre controlli $R M$ (Tabella 1). La percentuale FF presenta una correlazione statisticamente significativa con il numero delle piastrine $(r=0,72 ; p=0,01)$, mentre non è stata osservata nessuna correlazione statisticamente significativa tra la FF e il numero dei globuli rossi, dei globuli bianchi, dei neutrofili, dei lin- 


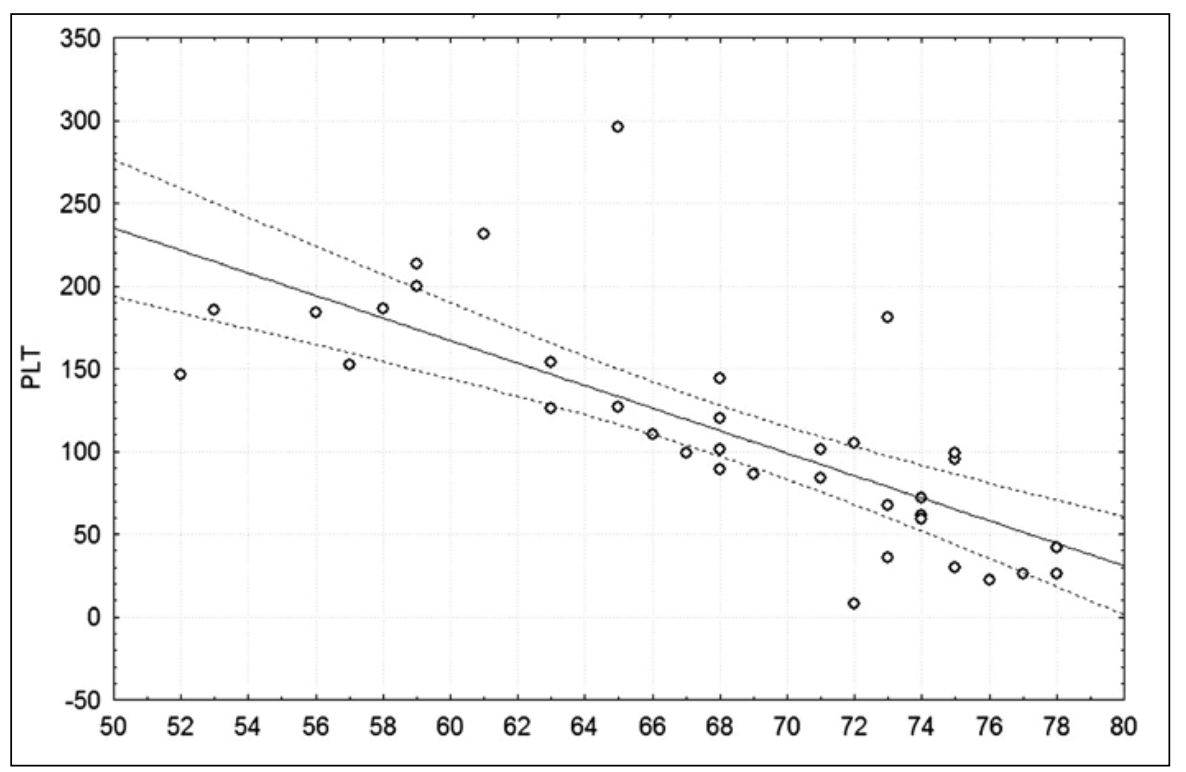

Fig. 5 Good correlation between values of FF\% and blood platelets $(r=-0.74 ; p<0.001)$.

Fig. 5 Il grafico mostra una buona correlazione tra $i$ valori di $F F \%$ ed il numero delle piastrine in circolo $(r=-0,74 ; p<0,001)$. ings of the two observers was $-1.2 \% \pm 4.2 \%$ with $95 \%$ limits of agreement, as estimated according to the Bland-Altman method (Fig. 6), between $-9.42 \%$ and $7.03 \%$. Differences in FF greater than the repeatability coefficient, i.e. $>8.4 \%$ $(p<0.05)$, observed in the same patient at the various time points may be considered statistically significant (or not due to interobserver variability).

Interobserver concordance coefficient Rho-C, estimated according to Lin's method, was $0.95 \pm 0.01$, with $95 \%$ confidence intervals $(\mathrm{CI})$ between 0.92 and $0.97(p<0.001)$.

The mean of the differences between the two FF recordings in the single patients was $1.11 \pm 3.75 \%$ in the first observer and $0.50 \pm 3.49 \%$ in the second, with $95 \%$ limits of agreement between $-6.23 \%$ and $8.46 \%$ for the first observer (Graph 1) and $-6.35 \%$ and $7.35 \%$ for the second (Graph 2). The coefficient of repeatability was $7.5 \%(p<0.05)$ for the first observer and $6.98 \%(p<0.05)$ for the second.

\section{Discussion}

The literature contains many studies on the application of MR imaging to patients with blood and lymph node diseases [11-15]. A study conducted by Schick et al. [6] states that MR imaging of the lumbosacral spine, in contrast to biopsy techniques that demonstrate only the local composition of the bone marrow, provides both a characterisation of the composition of the haematopoietic bone marrow and an estimation of the spatial distribution of water and fat, thereby enabling a global and noninvasive evaluation of the bone marrow and providing important additional fociti e dell'ematocrito (Fig. 5). La media delle differenze tra le rilevazioni di $\mathrm{FF} \%$ dei due osservatori è $-1,2 \% \pm 4,2 \%$ con limiti di accordo al 95\%, stimati secondo il metodo di Bland-Altman (Fig. 6), compresi tra -9,42\% e 7,03\%. Possono essere considerati statisticamente significative, ovvero non dovute alla variabilità inter-osservatore, differenze in FF\% maggiori del coefficiente di ripetibilità, ovvero maggiori del $8,4 \%(p<0,05)$, rilevate nel medesimo paziente in controlli successivi.

Il coefficiente di concordanza inter-osservatore Rho_C, stimato secondo il metodo di Lin è pari 0,95 $\pm 0,01$ con intervalli di confidenza al 95\% compresi tra 0,92 e 0,97 $(p<0,001)$.

La media delle differenze tra le 2 rilevazioni di FF\% nei singoli pazienti è pari a $1,11 \% \pm 3,75 \%$ nel primo osservatore e 0,50\% $\pm 3,49 \%$ nel secondo osservatore con limiti di accordo al 95\% compresi rispettivamente tra $-6,23 \%$ e $8,46 \%$ per il primo osservatore (Fig. 6) e tra-6,35\% e 7,35\% per il secondo osservatore (Fig. 5). Il coefficiente di ripetibilità del primo osservatore è $7,5 \%(p<0,05)$, mentre per il secondo osservatore è $6,98 \%(p<0,05)$.

\section{Discussione}

In letteratura vi sono diversi studi riguardanti l'applicazione dell'esame RM su pazienti con emolinfopatie [11-15]. Uno studio condotto da Schick et al. [6] asserisce che lo studio con RM del rachide lombo-sacrale, in contrasto con le tecniche bioptiche che dimostrano solo la composizione locale del midollo osseo, permette sia una caratterizzazione della composizione del midollo osseo emopoietico che una stima della distribuzione spaziale di acqua e lipidi, consentendo quindi una valutazione globale non invasiva del midollo osseo e for- 

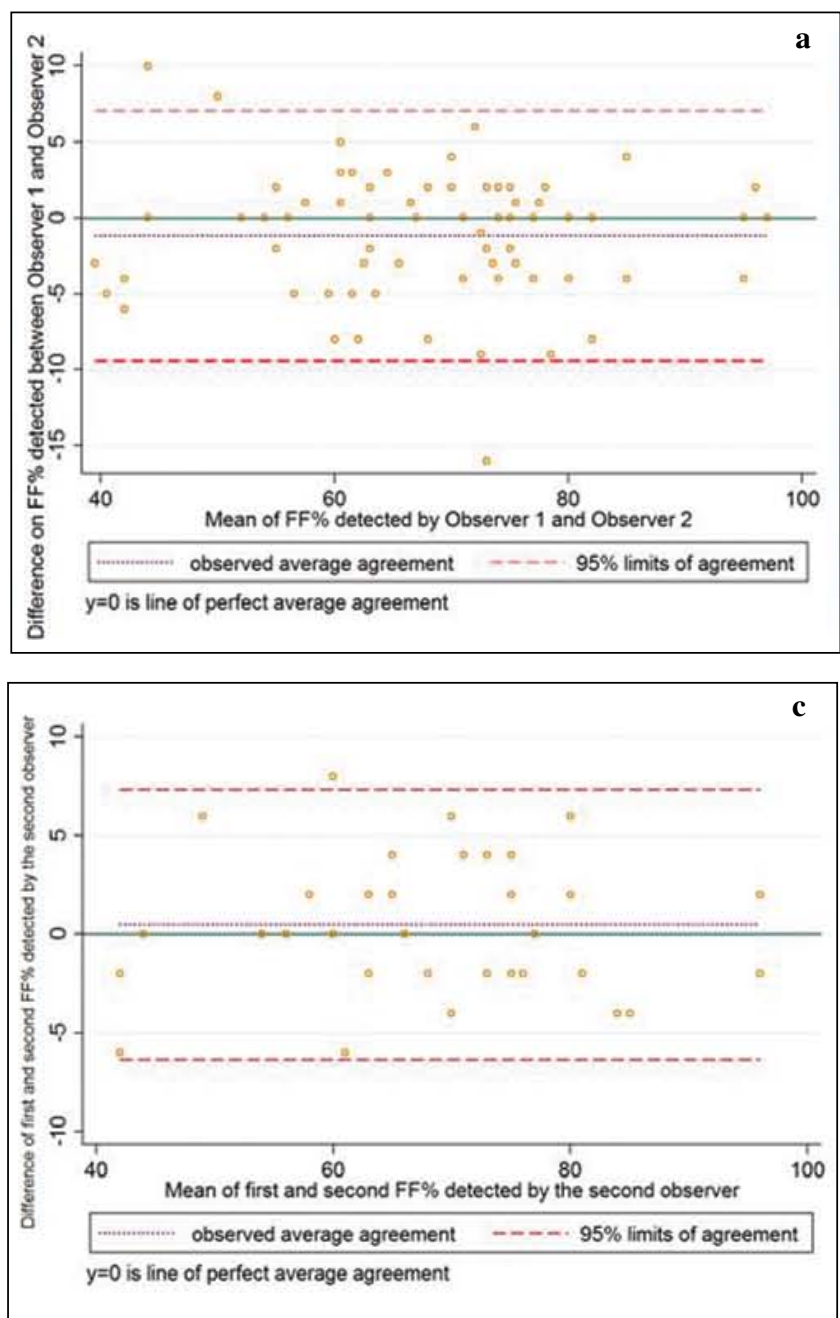

clinical information [6]. In fact, clinical and laboratory data - despite being abnormal in the event of blood and lymph node diseases - always need to be complemented with bone marrow aspiration or core biopsy in order to reach a definite diagnosis. Considering variability in disease site and extension, investigations performed at one site only may often lead to sampling errors in that they fail to provide diagnostic results representative of the entire haematopoietic system [16]. In addition to establishing the presence of disease, MR imaging can guide a biopsy towards a specific disease site and monitor, in a noninvasive manner, response to treatment through serial examinations.

MR imaging and MR spectroscopy of the bone marrow at sites with a greater amount of haematopoietic marrow (vertebrae and pelvis) provide fast and noninvasive estimations of the composition of bone marrow and the ratio between red and yellow marrow. This is made possible by the intrinsic characteristics of MR (i.e. signal intensity in T1 and T2) and chemical shift, that is, the different resonance

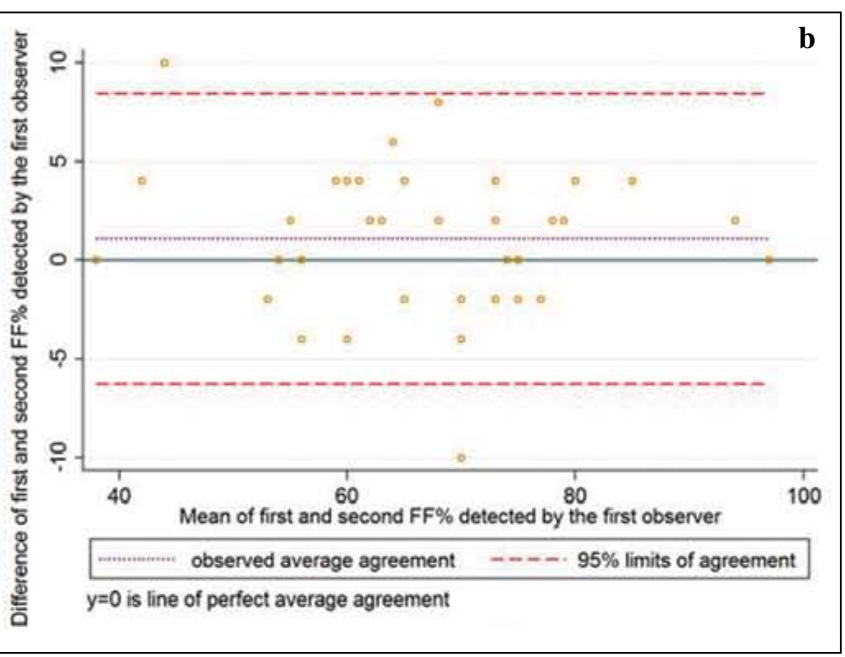

Fig. 6a-c Bland-Altman graph of inte-observer variability (a) and intraobserver variability $(\mathbf{b}, \mathbf{c})$. The straight line $(\mathrm{y}=0)$ indicates the hypothetical perfect agreement, whereas the dotted line represents the observed agreement. The two dashed lines indicate the limits of agreement. The single dots indicate the mean differences between the two observer's determinations.

Fig. 6a-c Grafico di Bland-Altman relativo alla variabilità inter-osservatore (a) ed intra-osservatore $(\boldsymbol{b}, \boldsymbol{c})$. La linea continua $(y=0)$ rappresenta la linea di concordanza perfetta, mentre la linea punteggiata rappresenta la concordanza osservata. Le 2 linee tratteggiate rappresentano gli estremi dei limiti di accordo. I singoli punti indicano le differenze medie tra le rilevazioni dei due osservatori.

nendo delle informazioni cliniche aggiuntive rilevanti [6]. In effetti, $i$ dati clinici e di laboratorio pur essendo anormali in presenza di emolinfopatia, necessitano sempre d'integrazione mediante ago aspirato o biopsia midollare per giungere a una diagnosi di certezza; considerando la variabilità in sede ed estensione di malattia, spesso tali indagini condotte effettuate in un solo punto, possono portare ad un errore di campionamento in quanto non consentono di ottenere risultati diagnostici rappresentativi di tutto il sistema ematopoietico [16]. La RM oltre a stabilire la presenza della patologia, può condurre in modo appropriato una biopsia verso una specifica localizzazione di malattia midollare e monitorizzare, in maniera non invasiva, la risposta della malattia al trattamento mediante controlli seriati nel tempo.

La RM e la spettroscopia con RM del midollo osseo nelle sedi in cui è più abbondante nell'adulto il contenuto di midollo osseo emopoietico (vertebre e bacino) permette di ottenere in maniera assolutamente non invasiva e in breve tempo una stima della composizione del midollo osseo stesso ed il rapporto midollo rosso/midollo giallo. Ciò è reso possibile grazie alle caratteristiche intrinseche della RM (cioè all'intensità del segnale rispettivamente in $\mathrm{T1} e$ in $\mathrm{T2}$ ) $\mathrm{e}$ so- 
frequency of hydrogen $(\mathrm{H})$ atoms according to the chemical bonds in which they participate. This way, it is possible to separate and quantify the metabolites present inside the VOI and obtain indirect estimations of the functional status of the haematopoietic red marrow.

More specifically, the spectrum obtained in a vertebra is mainly composed of a series of peaks: the first, between 0.8 and $2.2 \mathrm{ppm}$, belongs to the signal of the chemical bonds of the methylene groups $\left(-\mathrm{CH}_{2}\right)$, at $1.2 \mathrm{ppm}$ and methyl $\left(-\mathrm{CH}_{3}\right)$ at $0.8 \mathrm{ppm}$, contained in the aliphatic chains of saturated fatty acids; the second is at $5.6 \mathrm{ppm}$ and is generated by the vinyl groups $(-\mathrm{CH}=\mathrm{CH})$ contained in unsaturated fatty acids; finally, the signal of water $\left(\mathrm{H}_{2} \mathrm{O}\right)$ is located at $4.8 \mathrm{ppm}$. The percentage relationship between the total signal from lipids and that of water is defined as FF and provides an estimation of the composition of bone marrow and ratio between red and yellow marrow [17]. In fact, hypocellular bone marrow (yellow marrow) is histologically rich in lipids and low in haematopoietic cells, so that the spectrum will show a high FF due to limited water content. Conversely, a marrow rich in haematopoietic cells - therefore with a high density of stem cells (red marrow) will exhibit a spectrum with a low lipid content and a high water peak and therefore with a low FF. In haematological cancers (leukaemias, lymphomas, myelomas), the bone marrow is completely replaced by water-rich tumour cells, so the spectrum produced usually has a very low FF. After ablative therapy, the cellular component decreases, leaving virtually only a lipid peak, often associated with haemosiderin deposition due to cell death, with resulting magnetic field heterogeneity and major artefacts detrimental to spectral quality [18].

In our study, there was a statistically significant increase $(p<0.05)$ in FF values immediately after ablative therapy in 8/12 (67\%) patients who underwent BMT, thus reflecting cell loss, followed by a more-or-less slow marrow recovery shown by a reduction in fat content (Figs. 2-4). In the remaining four patients (33\%), there was a significant increase $(p<0.05)$ in FF values only at the second MR examination, suggesting a lower immediate efficacy of ablative therapy or incorrect timing of the examination. Considering all FF recordings - also in light of intraand interobserver variability in spectroscopic determinations - we saw a significant increase $(p<0.05)$ in FF values at the second MR examination in $100 \%$ of patients studied.

On the subject of inter- and intraobserver variability, some points need to be made. In particular, the value of interobserver concordance ( $\mathrm{Rho}-\mathrm{C}=0.95 \pm 0.1$ ) is very high, indicating a very high level of agreement between the two observers. However, the repeatability coefficient - equal prattutto al fenomeno del chemical shift, cioè alla diversa frequenza cui risuonano gli atomi d'idrogeno $(H)$ secondo $i$ legami chimici in cui sono incorporati. In tal modo è possibile separare e quantificare i metaboliti presenti all'interno del nostro volume d'interesse e avere stime indirette dello stato funzionale del midollo osseo ematopoietico.

Nello specifico, lo spettro ottenuto in una vertebra è composto prevalentemente da una serie di picchi: il primo, situato tra 0,8 e 2,2 parti per milione (ppm) appartiene al segnale dei legami chimici dei gruppi metilene (- $\left.\mathrm{CH}_{2}\right)$, a 1,2 ppm e metile $\left(-\mathrm{CH}_{3}\right)$ a 0,8 ppm, contenuti nelle catene alifatiche degli acidi grassi saturi; il secondo è situato a 5,6 ppm ed è generato dai gruppi vinilici $(-\mathrm{CH}=\mathrm{CH})$ contenuti negli acidi grassi insaturi, Infine il segnale dell'acqua $\left(\mathrm{H}_{2} \mathrm{O}\right)$ è situato a 4,8 ppm. Il rapporto percentuale tra il segnale totale fornito dai lipidi e quello fornito dall'acqua è definito FF\% e fornisce una stima della composizione del midollo osseo e del rapporto midollo rosso/midollo giallo [17]. Infatti, un midollo osseo ipocellulare (midollo giallo) è dal punto di vista istologico ricco in lipidi e povero di elementi cellulari emopoietici, per cui lo spettro mostrerà una FF\% elevata, per il basso contenuto idrico; di contro in un midollo ricco in elementi ematopoietici, quindi con un'alta densità di cellule staminali (midollo rosso), osserveremo uno spettro con un basso contenuto in lipidi a fronte di un picco dell'acqua elevato, quindi con una FF\% bassa. Nelle patologie oncoematologiche (leucemie, linfomi e mielomi) il midollo osseo è completamente sostituito da elementi neoplastici, ricchi in acqua, pertanto lo spettro ottenuto in questi casi mostra in genere una FF\% bassissima; dopo terapia ablativa diminuisce la componente cellulare per cui abbiamo quasi solo il picco dei lipidi, cui spesso si associa la deposizione di emosiderina, dovuta alla morte degli elementi cellulari, con conseguente disomogeneità del campo magnetico, che può provocare notevoli artefatti alla qualità dello spettro ottenuto [18].

Nel nostro studio abbiamo osservato un aumento statisticamente significativo $(p<0,05)$ dei valori di $F F \%$ in 8 dei $12(67 \%)$ nei pazienti sottoposti a trapianto di midollo osseo, subito dopo l'induzione della terapia ablativa, indice di un impoverimento cellulare, seguito da un più o meno lento recupero midollare, rivelato da una riduzione del contenuto lipidico (Figg. 2-4). Nei rimanenti 4 pazienti (33\%), si è osservato un incremento statisticamente significativo $(p<0,05)$ dei valori di FF\% solo nel secondo follow-up, suggestivo per una minore efficacia immediata della terapia ablativa o per un timing non corretto nell'esecuzione dell'esame. Considerando le rilevazioni di FF\% nel complesso, anche alla luce della variabilità intra ed inter osservatore nelle misure spettroscopiche, si è rilevato un incremento significativo $(p<0,05)$ di FF\% nel secondo follow-up nel 100\% dei pazienti studiati.

Per quanto concerne la variabilità inter- ed intra-operato$r e$, sono d'obbligo alcune precisazioni. In particolare il valore di concordanza inter-osservatore rilevato $($ Rho- $C=0,95 \pm 0,1)$ è molto elevato, indicando che mediamente $i$ due osservatori esiste un elevato di grado di concordanza nelle rilevazioni. Tuttavia il coefficiente di ripetibilità che ricordiamo essere 
to twice the standard deviation (SD) of the means of the differences between the two observers' determinations - is almost critical $(2 \times 4.2 \%=8.4 \%)$. This value should not be confused with the repeatability rate, as it indicates the values beyond which the differences between the two observers' determinations cannot be attributed to chance. Also, it is expressed as a percentage because in our study we quantified the percentage of free fats. As regards intraobserver variability, analysis of the graphs and values extrapolated showed that the second observer, although providing more consistent results (i.e. with lower mean differences between determinations), had very similar limits of agreement, so we can exclude any bias due to different levels of experience of the two observers. Instead, it might be interesting to investigate the repeatability of the determinations of the system for MR spectroscopic analysis in order to quantify the absolute errors on the single determinations provided by the machine.

Correlating FF values with cell types normally present in the full blood count, we found a significant correlation between FF and platelet count. This finding suggests that the number of circulating platelets after BMT provides the most accurate estimation of recovery of haematopoietic activity within the marrow or, in other words, of the efficacy of the BMT.

Given that in routine clinical practice the parameters used for establishing the timing of haematopoietic recovery after BMT are WBC and platelet counts. This correlation allows us to imagine a future role for MR spectroscopy both for monitoring BMT effectiveness and confirming poor or nonexistent residual marrow activity.

Our results are consistent with those of a study conducted by Shen et al. on patients affected by beta-thalassaemia treated with stem cell transplantation, which demonstrated that assessing the percentage of the area of red marrow in the proximal femur may be useful to predict the outcome of treatment and may be used as an additional predictive factor [19]. Schick et al. reported similar results, demonstrating that patients with low water content in the bone marrow have delayed haematopoietic reconstitution [6]. In contrast, Chabanova et al. failed to demonstrate that haematopoietic expansion monitored by MR spectroscopy correlates with the level of mobilised progenitor cells [7].

In conclusion, although our study lacked adequate statistical power due to the small population, it indicated that MR spectroscopy of the bone marrow in NHL patients treated with autologous stem cell transplantation is a noninvasive modality that is highly sensitive for characterising and monitoring bone marrow following transplantation. pari al doppio della deviazione standard delle medie delle differenze tra le rilevazioni dei due osservatori, assume un valore quasi critico $(2 \times 4,2 \%=8,4 \%)$. Questo valore non va confuso con la percentuale di ripetibilità, in quanto indica il valore oltre il quale le differenze tra le rilevazioni dei due osservatori non possono essere attribuite al caso ed è espresso in percentuale poiché in questo studio è stata quantificata la percentuale di grassi liberi. Relativamente alla variabilità intra-osservatore, dall'analisi dei grafici e dai valori estrapolati, possiamo osservare che il secondo osservatore pur fornendo risultati più consistenti, ovvero con una media delle differenze tra le rilevazioni appaiate, minore rispetto al primo osservatore, nel complesso ha limiti di accordo sovrapponibili e si può per tanto escludere la presenza di un bias dovuto alla differente esperienza tra gli osservatori. Invece potrebbe essere interessante indagare la ripetibilità delle rilevazioni del sistema di analisi spettroscopica RM in modo da poter quantificare in maniera precisa gli errori assoluti sulle singole rilevazioni della macchina.

Correlando $i$ valori di FF\% con gli elementi cellulari presenti normalmente nell'emocromo abbiamo ottenuto una correlazione statisticamente significativa tra $i$ valori di FF\% e il numero delle piastrine in circolo. Tale rilievo ci permette di ipotizzare che il numero delle piastrine in circolo dopo TMO è il parametro che ci dà una migliore stima della reale ripresa di attività ematopoietica midollare, cioè dell'efficacia del TMO.

Poiché nella routine clinica i parametri utili a stabilire $i$ tempi di attecchimento emopoietico dopo TMO, sono il numero dei globuli bianchi e delle piastrine in circolo, tale correlazione può permetterci di ipotizzare per la spettroscopia con RM un ruolo sia di monitoraggio dell'efficacia del TMO, sia di conferma della scarsa o nulla attività midollare residua.

I nostri risultati sono in accordo con uno studio condotto da Shen et al. [19] su pazienti affetti da beta-talassemia trattati con trapianto di cellule staminali, che ha dimostrato come la valutazione della percentuale della quantità di midollo rosso della porzione prossimale del femore può essere un utile parametro per prevedere la riuscita del trapianto e può essere utilizzato come un fattore predittivo addizionale [19]. Schick et al. [6] sono arrivati a risultati simili dimostrando che pazienti che hanno un basso contenuto idrico nel midollo osseo mostrano una ritardata ricostituzione midollare. Al contrario Chabanova et al. [7] avevano fallito nel dimostrare che l'espansione dei progenitori ematopoietici nel midollo osseo monitorizzata mediante RM spettroscopica correlava con i livelli di progenitori mobilizzati in circolo [16].

In conclusione, il nostro studio, pur richiedendo conferme più ampie poiché realizzato su una coorte relativamente piccola per fornire un'adeguata importanza statistica, ha indicato la RM con studio spettroscopico del midollo osseo in pazienti con LNH sottoposti a trapianto autologo di cellule staminali, come una metodica non invasiva e molto sensibile nella caratterizzazione e monitoraggio del midollo osseo dopo trapianto. 


\section{Conflict of interest None}

\section{References/Bibliografia}

1. Zinzani PL, Martelli M, Poletti V et al (2008) Practice guidelines for the management of extranodal non-Hodgkin's lymphomas of adult non-immunodeficient patients. Part I: primary lung and mediastinal lymphomas. A project of the Italian Society of Hematology, the Italian Society of Experimental Hematology and the Italian Group for Bone Marrow Transplantation. Haematologica 93:1364-1371

2. Barosi G, Carella A, Lazzarino $M$ et al (2006) Management of nodal diffuse large B-cell lymphomas: practice guidelines from the Italian Society of Hematology, the Italian Society of Experimental Hematology and the Italian Group for Bone Marrow Transplantation. Haematologica 91:96-103

3. Barosi G, Carella A, Lazzarino $M$ et al (2005) Management of nodal indolent (non marginal-zone) non-Hodgkin's lymphomas: practice guidelines from the Italian Society of Hematology, Italian Society of Experimental Hematology and Italian Group for Bone Marrow Transplantation. Haematologica 90:1236-1257
4. Bierman PJ, Phillips GL (2009) Role of hematopoietic stem cell transplantation in the treatment of non-Hodgkin's lymphoma. Cancer Treat Res 144:1-41

5. Steingass SK (2006) Hematopoietic stem cell transplantation in nonHodgkin's lymphomas. Seminars in Oncology Nursing 22:107-116

6. Schick F, Einsele H, Weiss B et al (1996) Assessment of the composition of bone marrow prior to and following autologous BMT and PBSCT by magnetic resonance. Ann Hematol 72:361-370

7. Chabanova E, Johnsen HE, Knudsen LM et al (2006) Magnetic resonance investigation of bone marrow following priming and stem cell mobilization. $\mathrm{J}$ Magn Reson Imaging 24:1364-1370

8. Bland JM, Altman DG (1986) Statistical methods for assessing agreement between two methods of clinical measurement. Lancet I:307310

9. Lin LI-K (1989) A concordance correlation coefficient to evaluate reproducibility. Biometrics 45:255-268

10. Lin LI-K (2000) A note on the concordance correlation coefficient. Biometrics 56:324-325

11. Ambrus CM, Ambrus JL (1975) Myeloid regeneration after whole body irradiation, autologous bone marrow transplantation, and treatment with an anabolic steroid. J Med 6:27-32
12. Crouch MA, Ross JA (1994) Current concepts in autologous bone marrow transplantation. Semin Oncol Nurs 10:12-19

13. Gribben JG, Nadler LM (1993) Bone marrow purging for autologous bone marrow transplantation. Leuk Lymphoma 11(Suppl 2):141-148

14. Roberts N, Smith SR, Percy DF et al (1991) The quantitative study of lumbar vertebral bone marrow using T1 mapping and image analysis techniques: methodology and preliminary results. Br J Radiol 64:673-678

15. Guglielmi G, Biccari N, Mangano F et al (2010) $3 \mathrm{~T}$ magnetic resonance imaging of the musculoskeletal system. Radiol Med 115:571-584

16. Schick F, Einsele H, Weiss B et al (1995) Characterization of bone marrow after transplantation by means of magnetic resonance. Ann Hematol 70:3-13

17. Fanucci E, Manenti G, Masala S et al (2007) Multiparameter characterization of vertebral osteoporosis with 3-T MR. Radiol Med 112:208-223

18. Machann J, Stefan N, Schick F (2008) (1)H MR spectroscopy of skeletal muscle, liver and bone marrow. Eur J Radiol 67:275-284

19. Shen J, Griffith JF, Cheng LN et al (2008) Bone marrow MR imaging as predictors of outcome in hemopoietic stem cell transplantation. Eur Radiol 18:1884-1891 